QL

435

.A1

086

1891

INVZ 


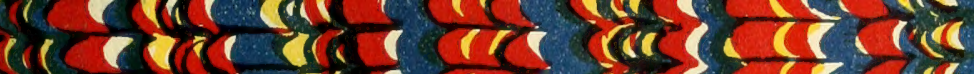

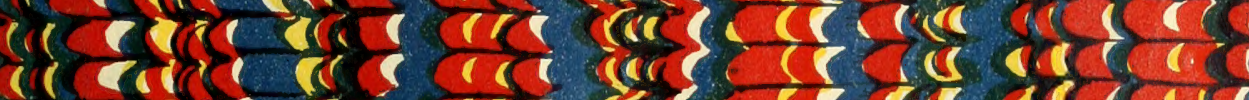

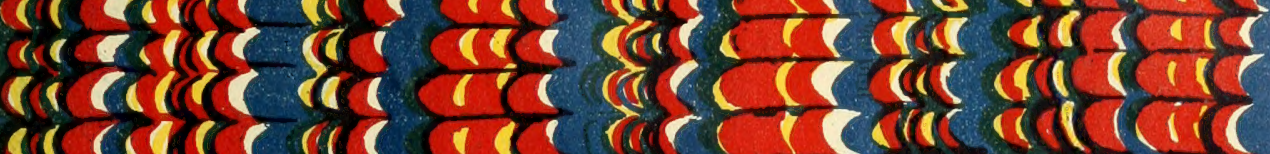

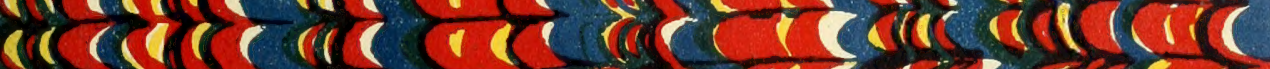

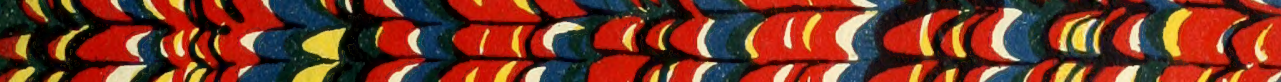

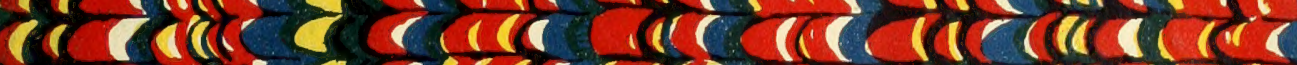

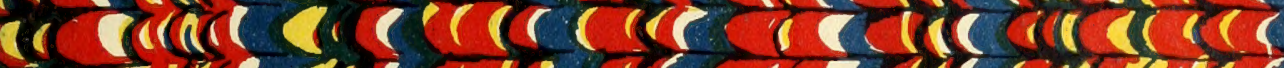

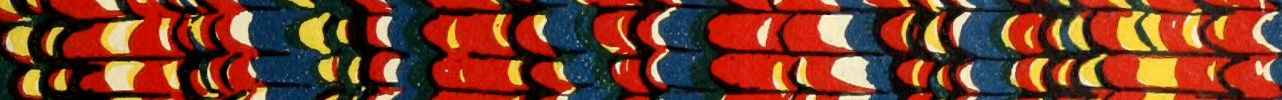
aceiger airs, is

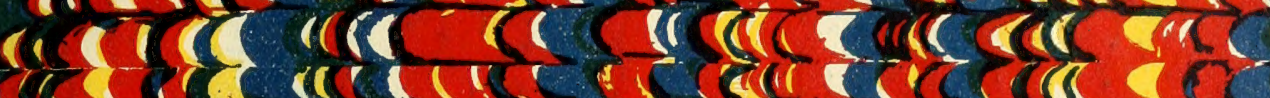

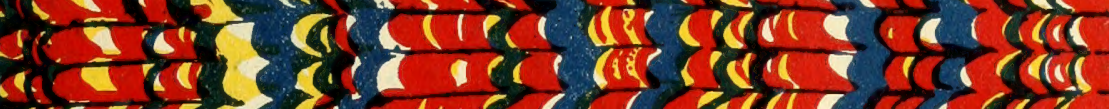
ercer तो ic किति वर्वा? i. कार र्यात ता रापार is (दाiि

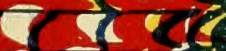
दि cerar rarec crivere

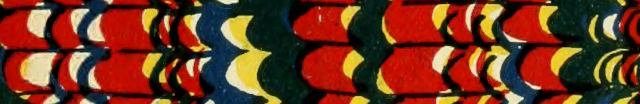

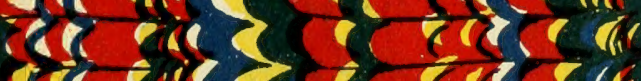

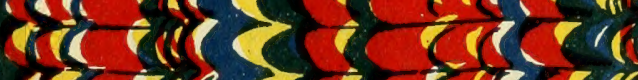
cer $>$ in arsing

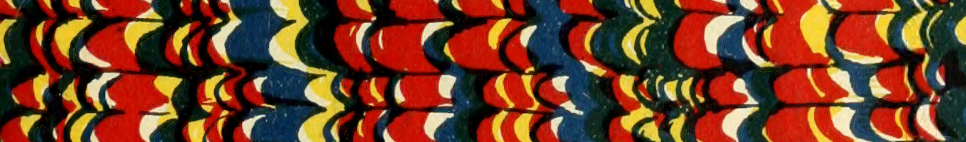
2

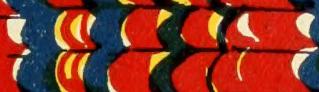
Theses $x+\frac{1}{2}$

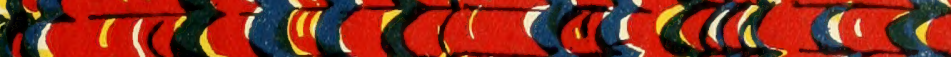

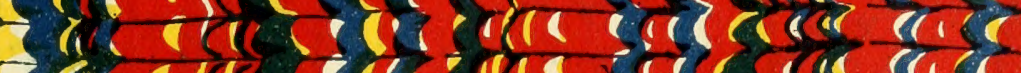

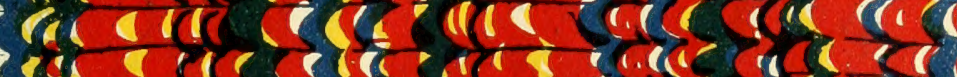

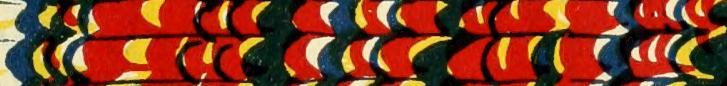

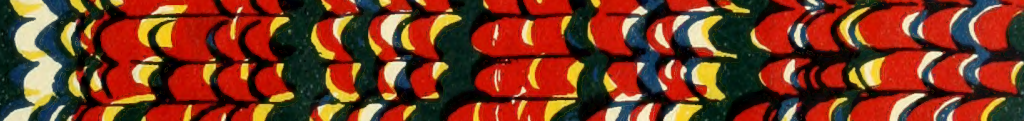

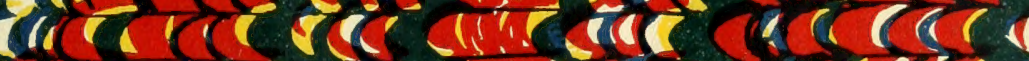

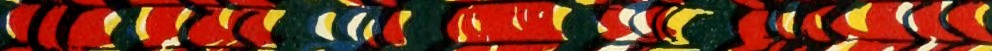
icrêtr rare

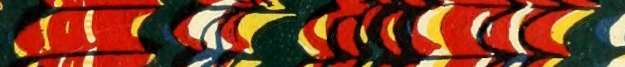

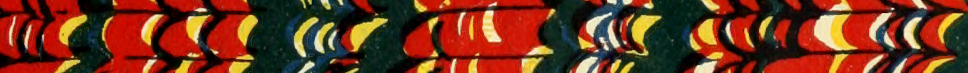

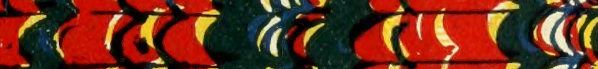
ता

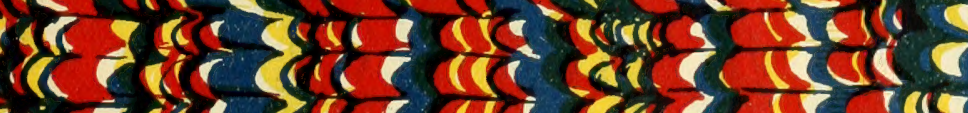

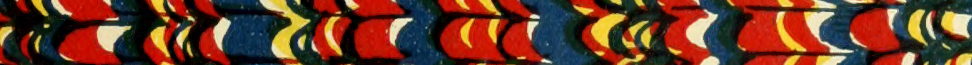

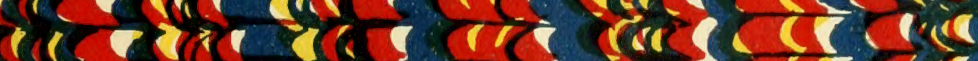

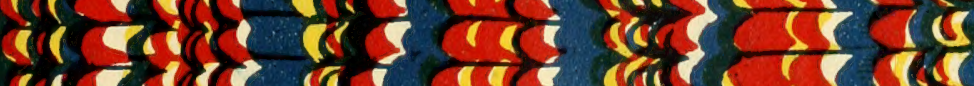

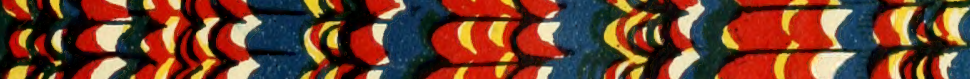

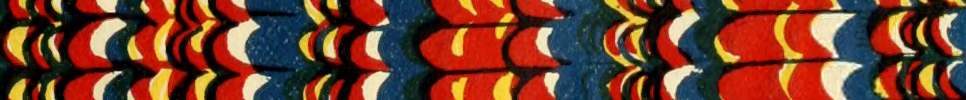

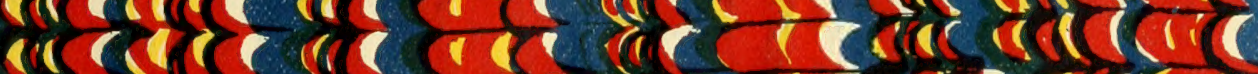

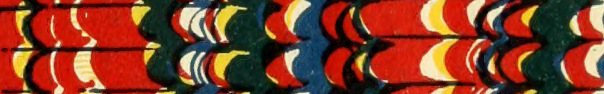
des cose $a$

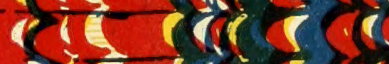
$-\mathrm{c}$ cere
cere risere

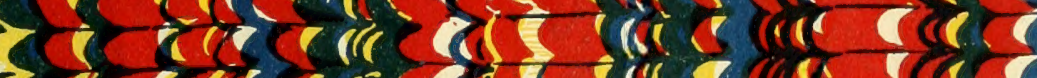
- कर 



Osario, Bathayar

Prustacea. (Binder'stitle).

244747 







I.
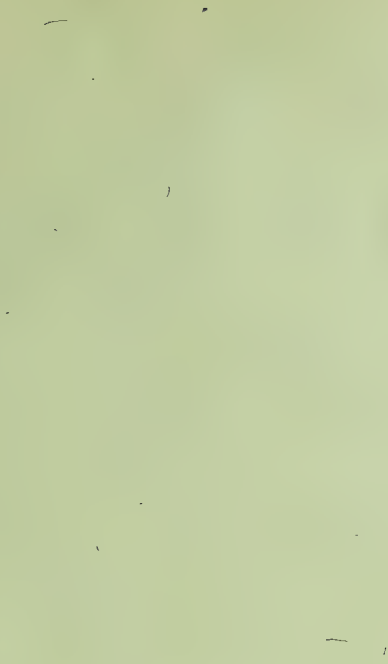

i 


$$
\because \because n \|
$$




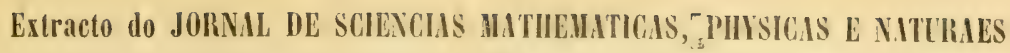
2. ${ }^{a}$ Serie - Tono VII - N.0 XXVI-Lisboa - 1904

\section{BREVE NOTICIA ÁCERCA DE ALGUNS PEIXES E CRUSTACEOS COLHIDOS NAS POSSESSÕES PORTUGUEZAS DA AFRICA OCCIDENTAL}

POI:

BALTHAZAR OSORIO

Entre os numerosos exemplares collidos pelo explorador portuguez F. Newton nas ilhas que compõem o archipelago de Cabo Verde encontram-se muitos peixes e crustaceos de agua doce e maritimos.

Algumas das especies são já conhecidas do Oceano Atlantico, mas a menção das que se inscrevem em seguida, assim como as de outras que incluiremos n'outra lista, é interessante porque circumscreve mais precisamente ou augmenta os limites da sua distribuição geographica.

Particularidades egualmente interessantes ácerca do habitat ou de caracteres especificos são tambem mencionadas.

Estas circumstancias motivam e justificam a publicação n'este jornal da presente nota, que é, em parte, complemento de outras já publicadas.

\section{Peixes das illas de Cabo Verde}

1. Holocentrum hastatum, Cuv, et Val.

Gunther, Cat. Fish. Brit. Mus., t. I, p. 39.

Habitat: Ilheo Raso (Cabo Verde).

N'este exemplar nota-se o seguinte: não existem as manchas esJехно, 1904. 
curas a que o prof. Gunther se refere na sua diagnose, a mancha negra que deve existir entre os primeiros espinhos dorsaes e entre os ultimos tres raios. E notavel, porém, que na estampa LIX da Historia natural dos peixes de Cuvier, em que esta especie vem representada, as manchas não existem, estando n'este ponto em perfeita harmonia com o que se vê no nosso exemplar.

As barbatanas peitoraes são amarellas pela sua parte interna (exemplar conservado em alcool), destacando-se da côr vermelha carmezim do corpo do peixe.

2. Serranus taeniops, Cuv. et Val.

Gunther, Cat. Fish. Brit. Mus., t. x, p. 121.

Habitat: ilhas de Cabo Verde (Ilheo Raso?).

3. Gerres melanopterus, Blkr.

Poiss. de Guiné, p. 44, pl. VIII, fig. 1.

Os exemplares d'esta especie que o sr. F. Newton enviou de Cabo Verde ao Museu de Lisboa foram colhidos em agua salobra (mistura de agua docc e agua do mar) na Boa Vista, o que confirma a idéa de que esta especie se não encontra exclusivamente nas costas dos mares tropicaes.

4. Smaris melanurus, Cuv, et Val.

Gunther, Cat. Fish. Brit. Mus., t. I, p. 389.

Habitat: ilhas de Cabo Verde (Ilheo Raso?).

\section{Gen. ĠIRELLA, Gray}

\section{Girella punctata? Gray.}

Girella punctata, Gray, Ill. Ind. Zool., pl. XCVIII, fig. 3 e 4; Melanichthys, Temm. et Schleg., Faun. Japon. Poiss., p. 75, pl. XXXIX; Gicella punctata. Gunther, Cat. Fish. Brit. Mus., t. I, p. 427.

Depois de estudo attento do exemplar que nos foi enviado pelo вr. F. Newton das ilhas de Cabo Verde não nos ficou duvida alguma de que o genero Girella existe no Oceano Atlantico e que não existe apenas nos mares da China e da Australia como se diz no Catalogo de Gunther.

E realmente um exemplar de Girella punctata que temos pre- 
sente? Julgamos que sim, pois as pequeninas divergencias que existem entre os caracteres que se observam nos nossos exemplares e os que sào apresentados nas diagnoses de Temm., Schlegcl e Gunther provêm talvez de uma observação feita em más condições. Por excmplo, Gunther diz que os dentes superiores sio dispostos em tres series e os inferiores em duas. No nosio exemplar contam-se quatro fiadas horizontaes de dentes na maxilla superior e tres na inferior. Os exemplares observados por Gunther sàr conservados a sececo, pelo que se deprehende do catalogo, e por este motivo os labios resequidos po. dem encobrir talvez fiadas de dentes difficeis de observar em semelhantes condições.

Temm. e Schlegel dizem na sua diagnose que os dentes da primeira fiada são maiores que os da segunda, e que os da terceira são dispostos em carda. Os dentes do nosso exemplar em qualquer das fiadas são todos de egual tamanho.

Por estas pequeninas divergencias e outras de somenos importancia deve crear-se uma especie nova? Parece-nos que não, e portanto inscrevemos o nosso exemplar sob a designação acima escripta.

6. Sargus Rondeletii, Cuv. et Val.

Gunther, Cat. Fish. Brit. Mus., t. I, p. 440.

Habitat: Ilheo Raso. Ilhas de Cabo Verde.

7. Sargus fasciatus, Cuv, et Val.

Hist. Nat. des Poiss., t. vi, p. 59; Gunther, Cat. Fish. Brit. Mus., t. r, p. 448.

Habitat: ilhas de Cabo Verde (Ilheo Raso).

Quem observar exemplares como o nosso melhor dirá talvez que é negro acobreado (exemplar conservado em alcool) com faixas brancas do que branco com faixas escuras, como diz Gunther. $O$ individuo que temos presente mostra seis faixas brancas, algumas das quaes vão um pouco além da linha lateral, e duas sómente, as da regiào caudal, a percorrem em toda a sua extensào. Querendo admittir o que diz Gunther relativamente á existencia de faixas cscuras sobre um fundo branco deve todavia entender-se pelo menos, attendendo an que se vî no nosso exemplar, que as faixas escuras são tres vezes mais largas do que as faixas brancas.

Gunther descreve apenas um unico exemplar.

Nenhum dos auctores a que nos referimos acima cita esta especie como proveniente das ilhas de Cabo Verde.

\section{Dactylopterus volitans, L.}

Gunther, Cat. Fish. Brit. Mus., t. Ir, p. 221.

Habitat: ilhas de Cabo Verde (Ilheo Raso?). 
9. Caranx ascensionis, Osbeck.

Gunther, Cat. Fish. Brit. Mus., t. II, p. 432.

Habitat: ilhas de Cabo Verde; Santo Antão.

No nosso exemplar não se nota simplesmente uma palpebra posterior, como diz Gunther e como se vê representado na tigura de Cuvier. Vê-se tambem uma palpebra anterior, embora não tão desenvolvida como a posterior.

10. Argyreiosus setipinnis, Gunth.

Gunther, Cat. Fish. Brit. Mus., t. II, p. 457.

Habitat: Ilheo Raso.

11. Lichia glauca, Linn.

Gunther, Cat. Fish. Brit. Mus., t. x, p. 456.

Habitat: Ilheo Raso.

12. Antigonia capros, Lowe.

Gunther, Cat. Fish. Brit. Mus., t. II, p. 487.

Habitat: ilhas de Cabo Verde; Santo Antão?

O exemplar que temos presente corresponde precisamente á curtissima diagnose d'esta especie apresentada por Gunther, com excepção apenas de um caracter que não nos parece ter grande importancia, dada a variação de numero de raios lanto da dorsal como da caudal que em tantas especies se verifica. Encontramos 37 raios e não 34 na barbatana dorsal, como diz Gunther.

Ias este celebre ichthiologista não viu nenhum exemplar d'esta especie, ajuiza pelas memorias de Lowe, MLull. e Trosch, que de resto não estão de accordo emquanto ao numero de arcos branchiaes.

Nào conhecemos nem a figura nem as memorias a que alludimos, mas julgamos estas em presença da especie cujo nome indicamós acima e que até agora era apenas conhecida dos mares da Nadeira e das Barbadas.

\section{Blennius sanguinolentus, Pall.}

Zool., IIr, p. 168; Blennius palmieornis, Cuv. et Val., t. xr, p. 214, pl. 320 Proc. Zool. Soc., 1839, p. 83 e 1843; Gunther, Cat. Fish. Brit. Mus., t. III' p. 218.

Habitat: Santa Luzia (Cabo Verde); Tlheo Raso. 
14. Clinus nuchipinnis, Quoy et Gaimard.

Gunther, Cat. Fish. Brit. Mus., t. II, p. 262.

Habitat: Ilheo Raso (Cabo Verde).

15. Glyphidodon saxatilis, Lin.

Gunth., Cat. Fish. Brit. Mus., t. IV, p. 35.

Habitat: Ilheo Raso.

16. Glyphidodon chrysurus, Cuv, et Val.

Gunther, Cat. Fish. Brit. Mus., t. Iv, p. 57.

Habitat: Ilheo Raso.

17. Clarias anguilaris, $\mathrm{L}$.

Gunther, Cat. Fish. Brit. Mus, t. 1v, p. 14.

Habitat: ilhas do Cabo Verde.

18. NIonacanthus scriptus, Osbeck.

Gunther, Cat. Fish. Brit. Mus., t. vin, p. 252.

Habitat: Cabo Verde.

19. Galeus canis, Rondelet.

De Pisc., p. 337; Galeus vulgaris, Yarrell., Brit. Fish., t. Ir, p. 491; Galeus canis, Bonap., Faun. Ital. Pesc.; Bocage e Capello, Peixes Plagiost., p. 18; Gunther, Cat. Fish. Brit. Mus., t. vi1, p. 379.

Habitat: Ilha de Santa Luzia.

$\mathrm{Um}$ individuo novo.

\section{Peixes da Guine}

Periophthalmus koelreuteri, Pall. var. $\alpha$.

Periophthalmus Papilio, Bl., Gunther, Cat. Fish. Brit. Mus., t. 1I, p. 99.

Habitat: Guiné. 


\section{Crustaceos das ilhas de Cabo Verde}

Atya scabra, Leach.

Habitat: Ribeira Brava, na Ilha de S. Nicolau (Cabo Verde). Palemon jamaicensis, Herbst.

Habitat: Ribeira Brava, Ilha de S. Nicolau (Cabo Verde). 







$m q^{1525224}$

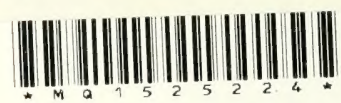

\section{Please add to PICKUST}

